\title{
Homenaje y agradecimiento a nuestros revisores
}

\author{
A tribute and thanks to our reviewers \\ Darío Echeverri* \\ Editor en Jefe, Revista Colombiana de Cardiología, Bogotá, Colombia
}

"The strongest arguments prove nothing so long as the conclusions are not verified by experience.

Experimental science is the queen of sciences and the goal of all speculation."

\section{(R. Bacon)}

Linkov F, Lovalekar M, LaPorte R. Scientific Journals are "faith based": is there science behind peer review? J R Soc Med. 2006;99:596-8.

Las revistas científicas continúan siendo la principal fuente de actualización y renovación de conocimiento, han sido fundamentales para el avance de la ciencia en los últimos tres siglos, y han hecho que esta exprese sus resultados y opiniones en la búsqueda de la verdad y nos permita, ante nuestros enfermos, tomar decisiones acertadas basadas en la mejor evidencia.

La Revista Colombiana de Cardiología ha venido evolucionando hacia un proceso de mejoramiento. Sin embargo, está enfrentada a grandes retos y amenazas, como la mayor disponibilidad de información a través de la Internet, las redes sociales y la proliferación de revistas predadoras. Estas condiciones universales ayudan a explicar el cambio radical que han sufrido la literatura y el aprendizaje médico en la última década.

Hoy, más que nunca, se hace necesario fortalecer nuestra Revista con artículos con mayor calidad científica, noticias nuevas y análisis crítico de la literatura disponible.
La palabra «revisión» es aparentemente simple, pero está cargada de una gran complejidad. Los revisores tienen una responsabilidad enorme para afrontar estos cambios radicales y mejorar nuestra publicación. "arte» de ejercer como árbitro requiere conocimiento, ética, imparcialidad, sensibilidad y mucha generosidad para orientar a los autores hacia un mejor manuscrito, de modo que se hagan visibles sus óptimos resultados. La posibilidad de una revisión por pares, adecuada y exigente, es un principio fundamental de las revistas científicas, y que debemos fortalecer, y por ende al método científico. Su propósito de mantener la objetividad, preservar la solidez de la ciencia, evitar conflictos de intereses y prevenir el fraude son características fundamentales de su ejercicio. Los revisores pares aseguran la calidad y la idoneidad para la publicación en la Revista; su buen obrar hace que nuestros lectores mantengan la credibilidad y la «fe» en su proceso de la publicación.

En este número queremos hacer un homenaje a nuestros revisores, profesionales distinguidos, altamente seleccionados, que ejercen su profesión en Colombia y en otros países y en quienes hemos depositado toda nuestra confianza por más de tres décadas de existencia. Gracias por su trabajo desinteresado, silencioso y valioso, base fundamental del proceso editorial de la Revista. Esperamos se afiancen en su proceder y continúen con mucha pasión, disciplina y responsabilidad como revisores para el bien de la ciencia.

\section{Correspondencia:}

*Darío Echeverri

E-mail: decheverri@lacardio.org
Disponible en internet: 22-02-2022 Rev Colomb Cardiol. 2022;29(1):1-2 www.rccardiologia.com 0120-5633 / @ 2022 Sociedad Colombiana de Cardiología y Cirugía Cardiovascular. Publicado por Permanyer. Este es un artículo open access bajo la licencia CC BY-NC-ND (http://creativecommons.org/licenses/by-nc-nd/4.0/). 
Nuestro agradecimiento sincero a nuestros revisores:

\begin{tabular}{|c|c|}
\hline Pedro Abad & Fernando Lizcano \\
\hline Claudia V. Anchique & Carlos A. Luengas \\
\hline Carlos Arias & Fernando Manzur \\
\hline Dagnóvar Aristizábal & Jorge E. Marín \\
\hline Julián M. Aristizábal & Sandra Matiz \\
\hline Álvaro Avezum & Enrique Melgarejo \\
\hline José R. Azpiri & Iván Melgarejo \\
\hline Ana M. Barón & Óscar Mendiz \\
\hline César Barrera & Fernán Mendoza \\
\hline Daniel Berrocal & Néstor Mercado \\
\hline Juan C. Briceño & Carlos Mestres \\
\hline Andrés F. Buitrago & Jorge D. Mor \\
\hline John Bustamante & Guillermo Mora \\
\hline Jaime Cabrales & Carlos Morillo \\
\hline Lina Caicedo & Ana Múnera \\
\hline Víctor Caicedo & Jaime Murillo \\
\hline Jaime Camacho & Solon Navarrete \\
\hline Paul Camacho & Federico Núñez \\
\hline Ivonne J. Cárdenas & Luz A. Ocampo \\
\hline Jonathan Cardona & Paola L. Páez \\
\hline Marisol Carreño & Ricardo Peña \\
\hline Carlos Carvajal & Nelson Pérez \\
\hline Gabriel Cassalett & Iván D. Rendón \\
\hline Mauricio Cassinelli & Jairo A. Rendón \\
\hline Roque A. Córdoba & Fanny Rincón \\
\hline Gina Cuenca & Mónica Rincón \\
\hline Tomás Chalela & Nubia L. Roa \\
\hline Juan A. Delgado & Diego Rodríguez \\
\hline Gabriel Díaz & Nohora I. Rodríguez \\
\hline José F. Díaz & Miguel Ronderos \\
\hline Juan C. Díaz & Fernando Rosas \\
\hline Mónica Duarte & Álvaro Ruiz \\
\hline Mauricio Duque & José F. Saaibi \\
\hline Pedro Forcada & Juan M. Sarmiento \\
\hline Argemiro Fragozo & Jaime A. Serna \\
\hline Ángel A. García & Pilar Serrano \\
\hline Efraín Gómez & Alberto Suárez \\
\hline Juan E. Gómez & Adriana Torres \\
\hline Mabel Gómez & Miguel Urina \\
\hline Nathalia González & Edgar Varela \\
\hline César Hernández & Diego Velásquez \\
\hline Édgar Hernández & Jorge Velásquez \\
\hline Heinz Hiller & Óscar Velásquez \\
\hline Andrés Iñiguez & Sebastián Vélez \\
\hline Nicolás Jaramillo & Boris Vesga \\
\hline Alejandro Jiménez & Ricardo Zalaquett \\
\hline
\end{tabular}

